\title{
Diverse Antioxidant Activity of Two Similar Cosmetic Emulsions Containing The Same Percentage of Green Tea Extract: The Former Contains the Alcoholic Extract And the Latter the Acqueous Extract obtained Via Ultrasound.
}

\author{
Lorenzo Martini, ${ }^{1}$ César Bimbi ${ }^{2}$ \\ ${ }^{1}$ Department Of Pharmaceutical Biotechnologies, University Of Siena, ${ }^{2}$ Member Of Dermatological Comittee Of \\ State Medical Council Of Rio Grande Do Sul Brazil, Brazilian Society Of Dermatologyv
}

\begin{abstract}
As it is well acquainted that the ultrasound extraction trials have demonstrated improvements in extraction yield ranging from 6 to 35\%, in this study I want to show the different antioxidant capacities of two cosmetic emulsions that contain the same percentage of a vegetable extract (e.g. Camelia sinensis, alias green tea), even if I tested the antioxidant power of the former cream that contained the alcoholic extract (presenting an antioxidating activity equivalent to 0.117) and of the latter cream that contained the same percentage of the green tea extract, obtained by Ultrasound method and the result is quite interesting: the antioxidant power of the latter creami s $58.20 \%$ higher than the former provides.
\end{abstract}

Keywords: Camelia sinenis extracts, Ultrasound extraction,polyphenol, UV absorbance, antioxidant activity.

\section{Background}

It must be stressed that Ultrasonic radiation is a powerful aid in accelerating various steps of the analytical but even of the extracting process in food industry and in manufacture of fragrances or herbalist's items. This energy is of great help in the pre-treatment of solid samples as it facilitates and speeds up operations such as the extraction of organic and inorganic compounds from vegetable stuff.. Ultrasound extraction in cool water is an effective way to extract biological principles from different matrices in shorter times than with other extraction techniques. For example, ultrasound systems have been widely used to extract carotenoids from marigolds or gojy berries or carrots. Ultrasonic extraction (USE) is considered one of the simplest extraction techniques because it is easy to perform in common laboratory equipment (i.e. ultrasonic bath). In this method, the crushed sample is mixed with the suitable solvent (generally water) and placed into the ultrasonic bath, where the working temperature and extraction time are set (cool or room temperature is always preferable, because of the thermoloability of biological actives) The application of ultrasound extraction technology is an add-on step to the existing process with minimum alteration, application in aqueous extraction where organic solvents can be replaced with solvents generally recognised as safe (GRAS), reduction in solvent usage, and shorter extraction time.

The use of ultrasonic means for extraction purposes in high-cost raw materials is an economical alternative to traditional extraction processes, this being a demand by industry for a sustainable development. The main goals of modern bio-technology are polyphenols and carotenoids and preferably in the form of aqueous extraction system. The ultrasound extraction trials have demonstrated improvements in extraction yield ranging from 6 to 35\% [1]. Many studies have examined the stability of the biological compounds during ultrasound-extraction process. Herrero et al. evaluated the phenolic-compound decomposition when phenolics were subjected to microwave extraction, and sonication was performed in order to assess the type of energy that provides a lower degradation of the principles. The A. applied the method to two types of strawberries in order to demonstrate the applicability of the proposed method, which is much faster and results in less actives degradation than do others [2]. Therefore, recently it has been shown that UE offers higher phenolic compound recovery when compared to pressurized hot-water extraction methods. (3)

The accurate assessment of oxidative stress in biological systems is a problem for all investigators working on the role of free radical damage in disease. Numerous assays have been described to measure various free radical damage products or antioxidant status, and the plethora of available techniques attests to the fact that no ideal method is available. The concept of a single test that might reflect total antioxidant capacity is to be considered an "Eldorado".

A comprehensive assessment of oxidative stress would include the measurement of all of the antioxidant enzymes that are present in the matrix as well,that tend to alterate results, although this would be time consuming, expensive, and in some cases technically difficult.(4) 
Now, amongst the manifold techniques to measure antioxidant capacity of a biological substance, Trolox equivalent antioxidant capacity (TEAC) measures the antioxidant capacity of a given substance, as compared to the standard, Trolox.

Trolox is the 6-hydroxy-2,5,7,8-tetramethylchroman-2-carboxylic acid and is a watersoluble analog of vitamin E. It is an antioxidant like vitamin $\mathrm{E}$ and it is used in biological or biochemical applications to reduce oxidative stress or damage. Most commonly, antioxidant capacity is also measured using the ABTS(Decolorization Assay). Other antioxidant capacity assays which use Trolox as a standard include the diphenylpicrylhydrazyl (DPPH), or oxygen radical absorbance capacity (ORAC) and finally the ferric reducing ability of plasma (FRAP) assays (known as Folin Ciocolteau's method). The TEAC assay is often used to measure the antioxidant capacity of foods, beverages and nutritional supplements, meanwhile the DPPH and the Folin Ciocolteau's are preferred to investigate the "probable and approximated" antioxidant capacity of biological principles extracted from plants and herbs.

Since I retrieved a very interesting Polish paper that describes exaustively a colorimetric interpretation of the most probable total antioxidant capacity of some vegetal extracts (a method similar to the Folin Ciocolteau's one, but that is able to test the capacity to avoid the oxidation of a sample linolenic acid) and the extracts the AA keep on account are right some Camelia sinensis extracts (acqueous, alcoholic,glycolic and glyceric), I wanted to try to behold if I could obtain different results, using the same method but the extract of fresh green tea leaves obtained via Ultrasound in cool water, considering that the Polish AA tested the pure extracts and $\mathrm{I}$ had to use two emulsions that contain $1 \%$ of the extracts (both alcoholic and acqueous via Ultrasound) and not the pure extracts. (6)

The emulsions contain $1 \%$ of Green tea extract, so I had to consider that I had to perform the thest knowing that I was using a hundredth of the pure extract and then, in order to the calculate the final result, I had to multiply by 100 the value obtained.

It is well acquainted that almost all plants and herbs do contain polyphenols and polyphenols are not but secondary metabolites of the plants themselves and are generally involved in defense against ultraviolet radiation or aggression by pathogens. Recenltly ,the entire world researchers' attention has been focusing more and more on the potential health benefits of polyphenols as antioxidant both in dietary supplement and in cosmetic items. Epidemiological studies and associated meta-analyses strongly suggest that long term consumption of diets rich in plant polyphenols or topical usage of the aforesaid phenols offer protection against development of cancers, and protection even towards skin melanoma or the simple epidermis senescence (5), as far as dermatologiical province is involved.

The antioxidant properties of green tea extracts owing to the thiocyanate method(the Folin Ciocolteau's method) are evaluated by the antioxidant properties of certain substances on the basis of assaying the content of peroxides, created as a result of linolenic acid oxidation, with the use of colorimetric method of creating thiocyanate complexes with $\mathrm{Fe}+3$ ions. The $\mathrm{Fe}+2$ ions in the solution, originating from iron(II) chloride, are oxidised in the presence of peroxides to $\mathrm{Fe}+3$ ions that bind with thiocyanate ions, creating red iron thiocyanates, measurable by UV spectrometry, according to the nuance that corresponds to the real quantity of Iron II obtained by reduction of the solution of Folin Ciocolteau (made up with ([Fe(SCN)6 ] 3-).

The measurements by UV light at $\lambda=500 \mathrm{~nm}$, may display a series of nuances of colours that can represent the antioxidant power, that will be measured by solving the following equation:

$$
\mathrm{AAC}=\mathrm{Ab}-\mathrm{At}
$$

Where $\mathrm{AAC}$ is the antioxidant activity, $\mathrm{Ab}$ is the $\mathrm{UV}$ absorbance at $\lambda=500$ of the blank test, $\mathrm{nm}$ and At is the absorbance at, $\lambda=500$ of the given substance that has to be evaluated. The colour intensity of the solution attests (by the aids of a UV spectrometer) the quantity of $\mathrm{Fe} 3+$ and thus can give the measurement of peroxide concentration. The complex bindings of iron and thiocyanate are unstable and already after 30 minutes the colour begins to fade, which is triggered by the reduction of iron(III) to iron(II), so that measurements at UV spectrometer have to be performed during the half hour successive to the reaction of linolenic acid with a strong oxidising agent (Hydrogen Peroxide is commonly used).

The conducted measurement of the colour intensity of [Fe(SCN)6 ] 3- complexes allowed for quantitative assessment of the effectiveness of antioxidant properties of green tea extracts.

Table I shows the AAC of 5 types of extracts of fresh leaves of Camelia sinensis:

\begin{tabular}{|l|l|l|}
\hline Examined sample & $\begin{array}{l}\text { Absorbance of blank test at } \lambda \\
500\end{array}$ & Antioxidant property \\
\hline Acqueous extract -0.046 0.058 & -0.046 & 0.058 \\
\hline Alcoholic extract & -0.060 & 0.117 \\
\hline Glycolic macerate & -0.135 & 0.107 \\
\hline Alcoholic macerate & -0.047 & 0.104 \\
\hline Glycolic extract & -0.096 & 0.068 \\
\hline
\end{tabular}


The Polish AA (6) attested that analyzing the results provided that the method of extraction and the type of solvent used can satisfactorily determine the effectiveness of antioxidant properties of green tea extracts. Among the examined extracts the best antioxidant properties were exhibited by the alcoholic extract

\section{Materials And Methods}

As I wrote in the Background, I have followed the revisited Folin Ciocolteau's method and analysed two emulsions containing the same percentage of green tea extracts. The first was prepared by a famous Italian firm that can boast to employ only extracts obtained by Ultrasound in cool water and so the percentage of extract of green tea was obtained via Ultrasound in cool water. The second emulsion contained even $1 \%$ of alcoholic extract, and its AAC is known, thanks to the Polish paper (6). I followed the same procedure of Polish AA, and so I poured in a conical flask of $50 \mathrm{~cm} 3$ with ground glass socket the mixture containing $10 \mathrm{~cm} 3 \mathrm{of}$ $99.8 \%$ ethanol, $10 \mathrm{~cm} 3$ of phosphate buffer in $0.1 \mathrm{~mol} / \mathrm{dm} 3$ and $\mathrm{pH}=7,5 \mathrm{~cm} 3$ of deionised water and only afterwards I added the antioxidant substance $(1 \mathrm{~cm} 3$ ofthe emulsion containing the $1 \%$ of green tea fresh leaves extract obtained via Ultrasound in cool water) and linolenic acid and hydrogen peroxide (in such quantity so that its final concentration in the mixture was $0.02 \mathrm{~mol} / \mathrm{dm} 3$ ). During the successive 30 minutes, using the UV- spectrophotometer I measured the solution absorbance at wavelength $\lambda=500 \mathrm{~nm}$.

Inserting the value obtained in the aforesaid equation and resolving it, the final result was 0.00201, but I had to multiply this value by 100 (since the Polish AA used $1 \mathrm{~cm} 3$ of pure extract (of whichever sort) and I had a hundredth of extract in my sample to test (because I had to measure $1 \mathrm{~cm} 3$ of cream containing $1 \%$ of extract). So the final result was 0.201 , and this result demonstarates that a cream containing an acqueous extract obtained via Ultrasound reveals an antioxidant power $58.20 \%$ higher than the same cosmetic system (presenting the same concentration of excipients and water and preservatives etc) that contains $1 \%$ of the alcoholic extract of green tea freh leaves.

\section{Discussions}

As I declared in the Background, it is true that Ultrasound extraction in cool water of biological actives that could be thermolabile are always to be preferred to other types of extraction, especially when polyphenols, that are extremely delicate, are involved. An absoulte antioxidant activity $58.20 \%$ higher of whichever cream that boasts the presence of Camelia sinenis extract (alcoholic, aqueous,glycolic or glyceric) is most convenient and sincerely it can be affirmed that the antioxidant power reveals a magnificient importance as far as the dermal cosmetological approach is concerned, both to decelerate the occurrence of skin melanoma and prevent skin senescence.

\section{Aknowledgements}

It has been possible to conduct this research thanks to the cream containing Camelia sinensis aqueous (US) extract obtained via Ultrasound radiations in cool water, provided by Solimé srl, Reggio Emilia.

\section{References}

[1]. Vilkhu, K.; Mawson, R.; Simons, L.; Bates, D. Applications and opportunities for ultrasound assisted extraction in the food industry. Innov. Food Sci. Emerg. 2008, 9, 161-169.

[2]. Dobiáš, P.; Pavlíková, P.; Adam, M.; Eisner, A.; Beňová, B.; Ventura, K. Comparison of pressurised fluid and ultrasonic extraction methods for analysis of plant antioxidants and their antioxidant capacity. Cent. Eur. J. Chem. 2010, 8, 87-95.

[3]. Garcia-Salas P, Morales-Soto A, Segura-Carretero A, Fernández-Gutiérrez A.; Review Phenolic-Compound-Extraction Systems for Fruit and Vegetable Samples: Molecules; 2010, 15, 8813-8826;

[4]. Young P: Measurement of total antioxidant capacity: J Clin Pathol. 2001; 54(5): 339

[5]. Pandey KB, Rizvi SI: Plant polyphenols as dietary antioxidants in human health and disease; Oxid Med Cell Longev. 2009; 2(5): $270-278$.

[6]. 6)Sikora E, Ogonowski J: Study of antioxidant properties of green tea extracts: Chemik 2011, 65, 10, 968-973 